\title{
Functional Foods: Towards Improving Oral Health
}

\author{
Itzhak Ofek, ${ }^{1}$ Carla Pruzzo, ${ }^{2}$ and David Spratt ${ }^{3}$ \\ ${ }^{1}$ Department of Clinical Microbiology and Immunology, Sackler Faculty of Medicine, Tel Aviv University, \\ Tel Aviv 69978, Israel \\ ${ }^{2}$ DIPTERIS, University of Genoa, 16132 Genoa, Italy \\ ${ }^{3}$ Department of Microbial Diseases, UCL Eastman Dental Institute, London WC1X 8LD, UK
}

Correspondence should be addressed to Itzhak Ofek, aofek@post.tau.ac.il

Received 24 November 2011; Accepted 24 November 2011

Copyright () 2012 Itzhak Ofek et al. This is an open access article distributed under the Creative Commons Attribution License, which permits unrestricted use, distribution, and reproduction in any medium, provided the original work is properly cited.

The aim of this special issue is to provide a foundation on which further research for the development of compounds from food as possible candidates for improving oral health could be based. The maintenance of oral health can be invariably achieved by manipulating the oral microbiota toward a population of mixed species that is less likely to induce diseases such as gingivitis or caries. Dental caries and gingivitis are the most prevalent infectious diseases of humans and are due to the accumulation of dental plaque (a bacterial biofilm) on the tooth surface and at the gingival margin, respectively.

There is evidence that certain beverages and foods can protect against caries and gingivitis. However the use of foodstuffs (functional foods) as a starting point for fractionation of compounds that influence the composition of the oral microbiota to one that is beneficial or at least nonpathogenic has been the focus of intensive research [1]. An advantage of this approach is that such natural agents are likely to be nontoxic. Furthermore, the identified active components can be used as food supplements negating the necessity to adhere to a particular diet as discussed by Shmuely et al. [2]. Perhaps most important advantage of searching for such dietary agents is that approval of clinical trials is easier to obtain, as toxicity is usually not an issue. Interestingly, dietary agents often lack bactericidal activity but retain their ability to manipulate the oral microbiota by exhibiting other important properties for example antiadhesion or antibiofilm activities. Such agents may be expected to decrease the selection pressure placed on resistant strains and therefore reduce their emergence in biofilm communities.
The collection of manuscripts in this special issue is mainly composed of research articles and one review article. The latter highlights the importance of food intake with emphasis on the effect of Mediterranean diet on oral health, discussed by G. A. Scardina and P. Messina. All the research articles represent methodological studies by a coherent group of investigators (Investigators from 7 academic institutions and an industrial partner representing 5 countries (University of Pavia, University of Tel Aviv, University of Genoa, Göteborg University, Academic Centre for Dentistry Amsterdam, and the University of Verona as well as an industrial partner, Givaudan). The consortium and project were named NUTRIDENT and was a $€ 2.2 \mathrm{M}$ specific targeted research project entitled "Towards functional foods for oral health care-isolation, identification and evaluation of beverage and food components with anti-caries and/or anti-gingivitis activities." It was funded from within the Framework Programme 6 (FP6)—Thematic Priority 5: Food Quality and Safety.)

Seven plant and fungal homogenates and extracts (green and black tea, cranberry juice, raspberries, shiitake mushrooms, red chicory, and beer) were subjected to a number of microbiological assays broadly related to survival and/or fitness of specific oral bacterial species usually associated with the development of either gingivitis or caries. This is discussed by D. A. Spratt et al. In this study it was found that the low-molecular-mass (LMM) fractions of shitake mushroom and chicory homogenates had the most significant anticaries and antigingivitis associated activities of the seven tested. These included inhibition of bacterial growth, adherence, coaggregation, biofilm formation, biofilm integrity, 
and signal transduction. A significant find was that these two homogenates also inhibited proinflammatory cytokine production. Further in-depth studies by the investigators focusing on specific bacterial activities were carried out. Shiitake mushroom extract lowered the numbers of some pathogenic oral bacteria without affecting bacteria associated with oral health. This is demonstrated by L. Ciric et al. Moreover, the compounds in the LMM fraction from shiitake mushroom inhibited dentin demineralization usually caused by cariogenic bacteria and induced a shift in the microbiota to that associated with oral health; this is shown by E. Zaura et al. The LMM fraction in shiitake mushroom and chicory homogenates also inhibited the adverse induction of genes expression in the gingival $\mathrm{KB}$ cell line by gingivitis bacteria; this is exhibited by L. Canesi et al. Efforts to characterize the effect of the chicory and mushroom extracts revealed that the extracts induced elongation of gingivitis-associated bacteria, reminiscent to that induced by sublethal concentrations of quinolones and $\beta$-lactam antibiotics; this is discused by $\mathrm{C}$. Signoretto et al. These in vitro findings prompted clinical trials with the LMM shiitake mushroom fraction. In one trial the total counts of plaque bacteria in volunteers rinsing with mouthwash supplemented with LMM mushroom fraction were significantly reduced to levels similar to those in samples of volunteers rinsing with Listerine; this is proven by C. Signoretto et al.). In another trial it was found that mouthwash containing the mushroom fraction reduced the metabolic activity of dental plaque, suggesting anticariogenic potential of the fraction; this is demonstrated by P. Lingström et al. These in vivo effects are reminiscent to the outcome of a clinical trial whereby rinsing with a cranberry fraction caused significant reduction in total oral bacteria including mutans streptococci [2]. Indeed as confirmation, and as shown in this special issue, the cranberry fraction reduced metabolic activity of preformed Streptococcus sp. biofilm; which is shown by J. Babu et al.

A detailed characterization of the plant and fungal extracts and the fractions arising from these was carried out by M. Daglia et al. and attempts to identify the active component(s) were initiated. All seven plant and fungal extracts were found to contain polyphenols; relatively high amounts were detected in beer, cranberry, and green and black tea by M. Daglia et al. Chicory and shiitake mushroom homogenates and raspberry contained the highest amount of zinc. Further studies are planned to identify the active components in these extracts especially those in the LMM fractions exhibiting the most favourable activity for maintaining a community associated with oral health.

In summary, promising foods/beverages have been identified using a range of in vitro and in vivo methods. These homogenates, or fractions of them, could be used in a number of ways to improve oral health, perhaps in mouthwashes or chewing gum.

\section{Acknowledgments}

We would like to thank the authors for providing such high-quality articles for this special issue of the Journal of Biomedicine and Biotechnology. We sincerely hope that this collection of papers will prompt further research and development of functional foods or compounds derived from these to be supplemented into various foods and beverages or as rinses to improve oral health.

Itzhak Ofek

Carla Pruzzo

David Spratt

\section{References}

[1] M. Wilson, Food Constituents and Oral Health: Current Status and Future Prospectse, Woodhead Publishing Series in Food Science, Technology and Nutrition No. 172, 2009.

[2] H. Shmuely, I. Ofek, E. I. Weiss, Z. Rones, and Y. HouriHaddad, "Cranberry components for the therapy of infectious disease," Current Opinion in Biotechnology. In press. 

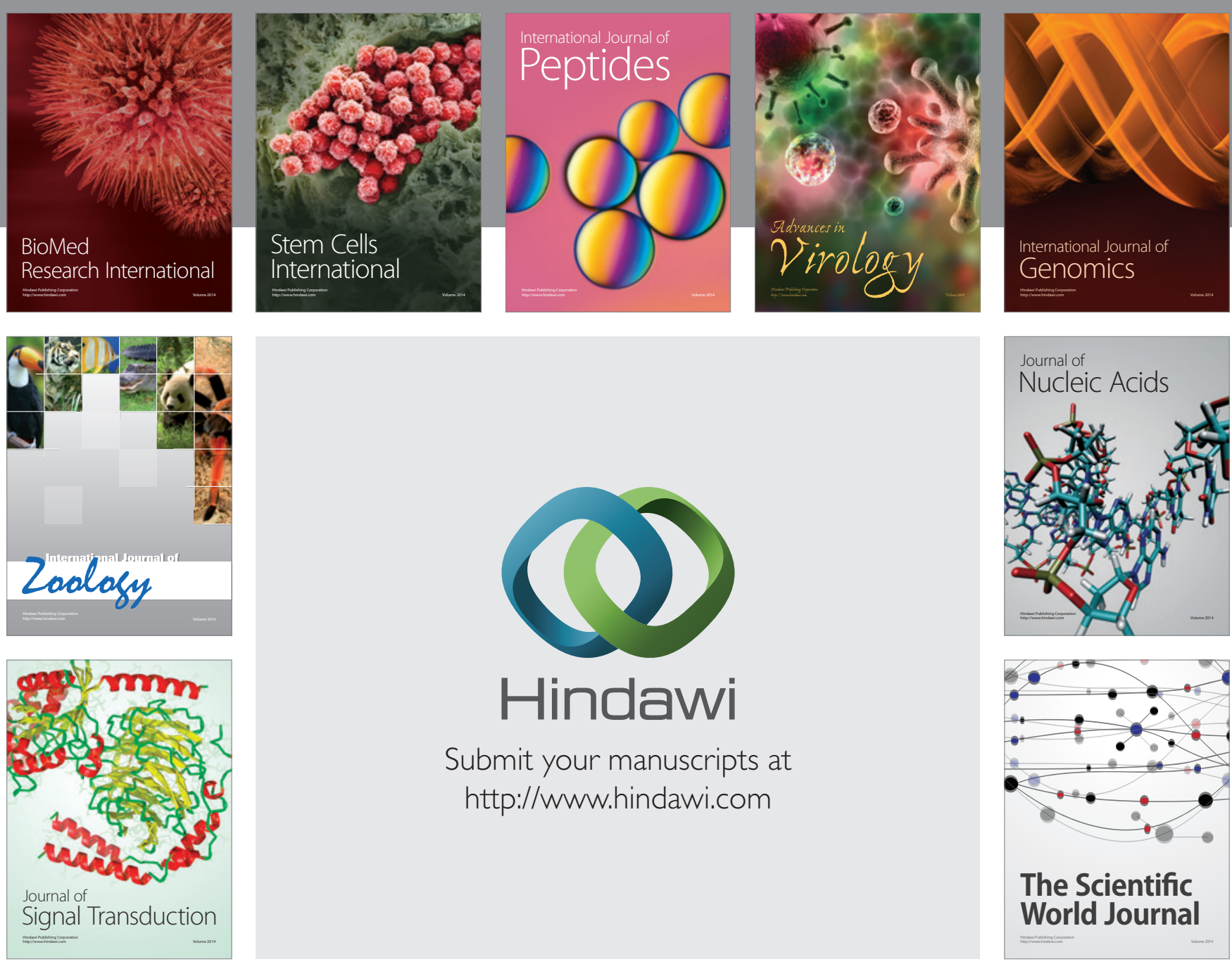

Submit your manuscripts at

http://www.hindawi.com
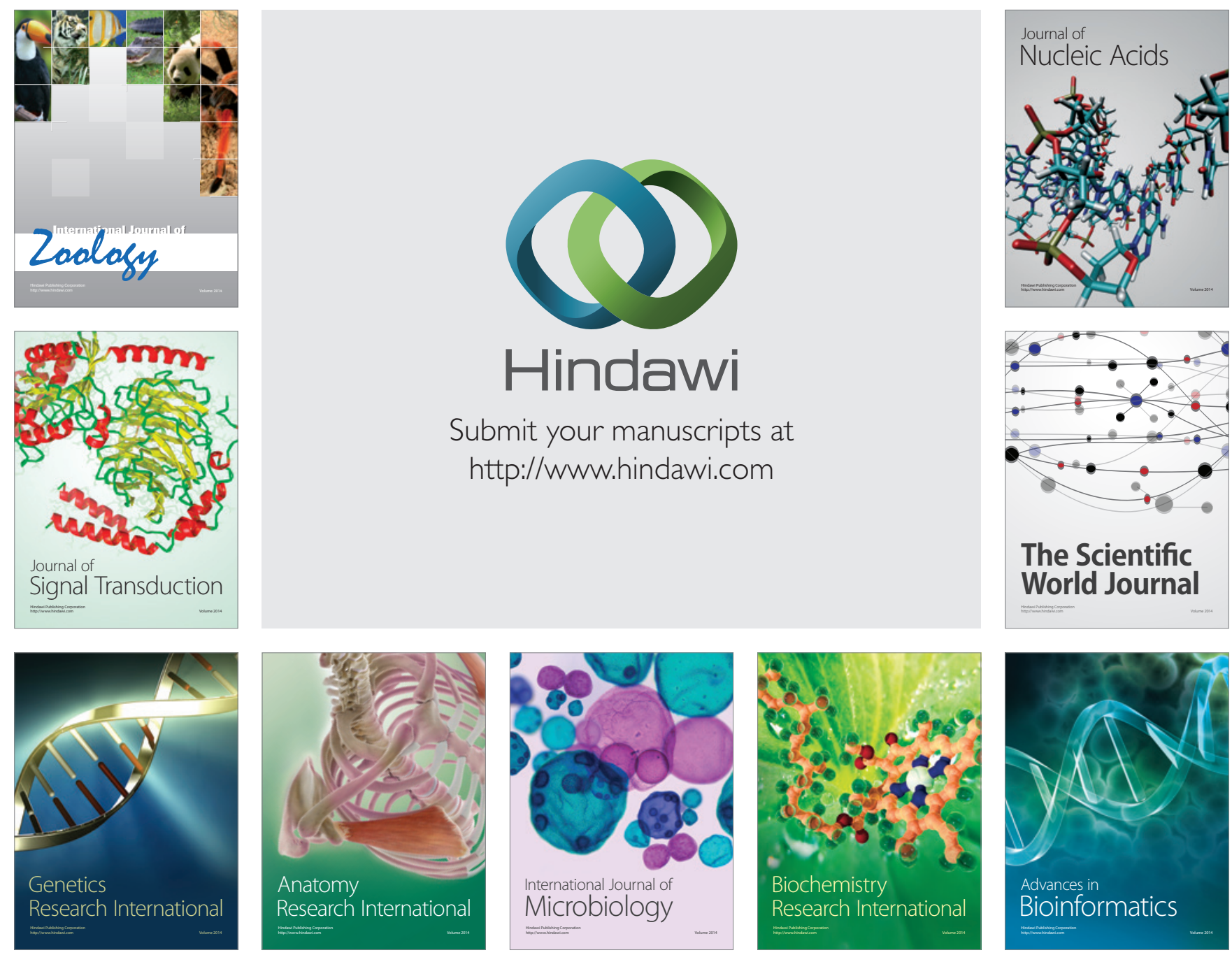

The Scientific World Journal
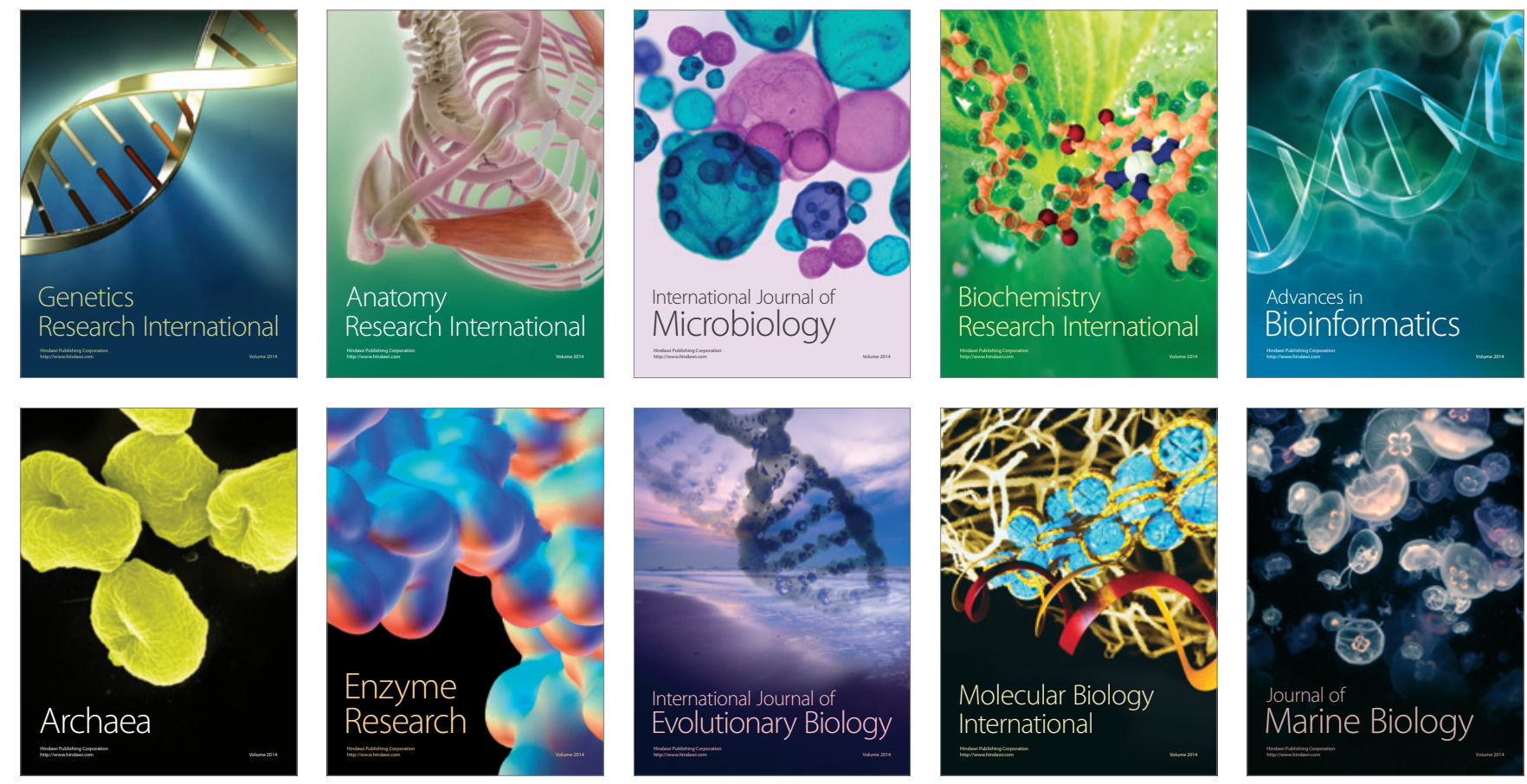